\title{
Review of: "Correlation of P-wave velocity with mechanical and physical properties of limestone with statistical analysis"
}

Jamal Khatib ${ }^{1}$

1 University of Wolverhampton

Potential competing interests: The author(s) declared that no potential competing interests exist.

Generally the paper contains a good set of data and analysis. The authors should highlight the novelty of the work and how it differs from similar research on this topic

Table 3 caption...location should it be on top of the table?

Figure 13 to Figure 25 . There are 23 figures with hardly any discussion.

English is generally good but requires improvement in some places. Examples are given below in square brackets [[ ]]

Seismic techniques are well understood, [[] non-destructive, and low-cost methods that can be easily performed in the laboratory and in-situ ]]]]]. 